\title{
IRIS based Color Image Compression
}

\author{
Asakti Rautkar \\ Asst. professor, Electronics and telecommunication Engineering, \\ Parvatibai Genba Moze College of Engineering, Pune, India \\ Dr. M. S. Ali \\ Principal \\ Prof Ram Meghe College of Engineering and Management, Amravati, India
}

\begin{abstract}
We put forward a Bio-inspired Image compression scheme which implies a combination of spiking retina model, as in a Human Visual System (HVS) with a well known Data compression technique. The crux of this work is to show how neural code can be exploited in the context of Image compression. The first stage being the bio-inspired retina model, which transforms an image into a wave of spikes [1 2 2]. Secondly edge mapping of edge mapping of the image with a mean coder and then Wavelet transform. Bio inspired computing being the most important and advanced field of research now a days . it basically consists of using the mechanism of the biological system to enhance the various aspects of computing.
\end{abstract}

Keywords - HUMAN VISUAL SYSTEM (Hvs), IRIS

\section{INTRODUCTION}

Images form the most significant form of data, especially in remote sensing, satellite communication, bio-medical application and video conferencing. As the dependence on information and computers continuous to grow so does our need to efficiently handle the data by storing and transmitting without loss [3]. Image compression is mapping of an image from higher dimensional space to low dimensional space, and it plays a vital role in multimedia application such as storage, transmission etc. The basic goal is to represent an image with minimal number of bits of an acceptable quality.

Compression is achieved by the removal of one or more of the three basic data redundancies:

- Coding Redundancy

- Inter pixel Redundancy

- $\quad$ Psycho visual Redundancy

Clubbing of the concept of compression with the human eye communication is done through the Bio-inspired computing method which is an up growing and important of research. It basically consists of employing the biological system to enhance the aspects of computing. The mammalian biological system is studied and based upon the natural phenomenon present an understanding and development of specialised output in accordance with nature is implemented.

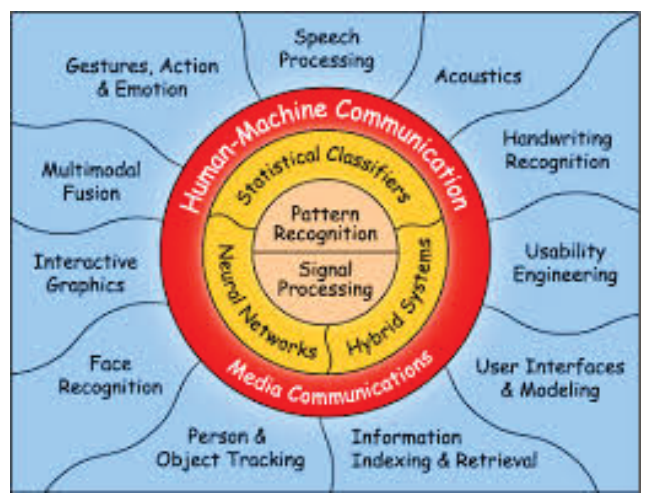

Fig 1: The Human machine communication outlook.

The human visual system conveys information as a set of electrical impulses called spikes and these are the way our nervous system chooses to communicate [4]. The image perception involves a complex set of procedures which when executed step by step lead to the exact concept of image read, the light intensity is the first and foremost part of the image acquiring, this light is taken by the receptor cells adjoining the inner periphery of the 
eyes then this signal is converted to electrical form and subsequently forwarded to the neurons by the ganglion cells, and finally to the brain through the optic nerve connecting the eye and the brain.

The path flow of our work is to read an image (colour or gray scale) convert it into individual Red, Blue or Green plane if necessary, then apply the IRIS model of the Human Visual System through the Rank order coding (ROC) [1 2] of the stimulus and process further by edge mapping to enhance its sharpness and then compressing the image without rendering the image, extracting the relevant data.

\section{SYSTEM MODEL}

Our compression scheme is implemented in 4 stages, first converting the colour image into respective R, G, B plane a spiking retina model. Secondly, a spiking retina model based on Rank order coding (ROC), edge detector and then Wavelet Transform for Compression.

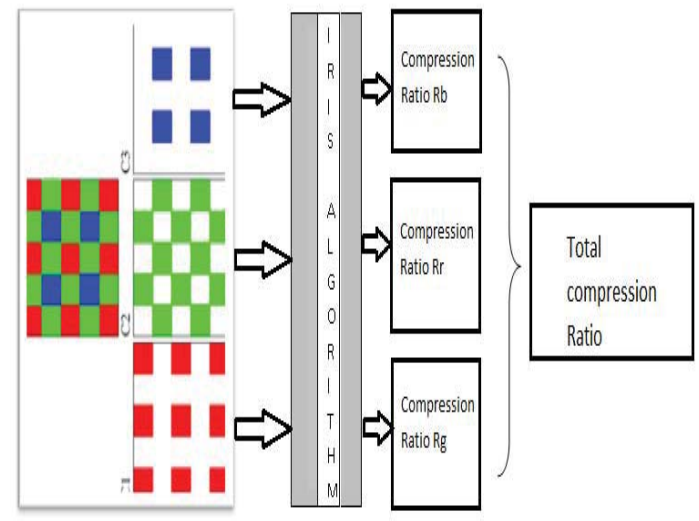

Fig.2. The Basic Idea of the Approach

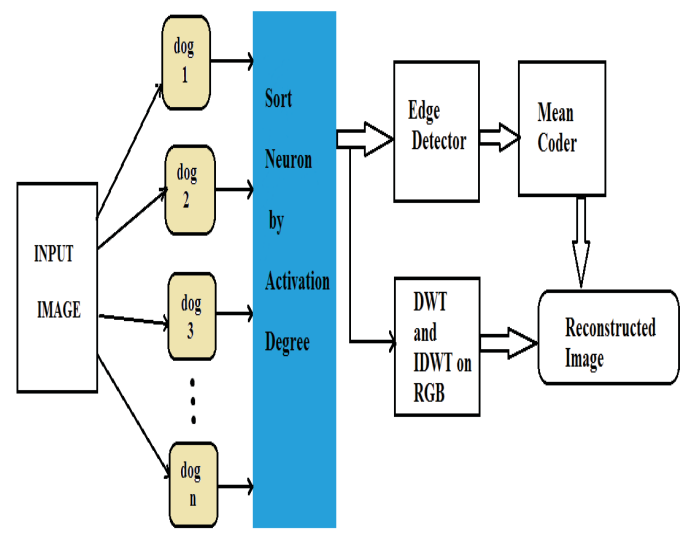

Fig 3: The IRIS model in working

\section{PREVIOUS WORK}

The previous two decades research in still image compression gave birth to several coding algorithms especially the JPEG effort which led to be a standard of the same name. Since then constant continuous efforts are being following the schema for image coding.

Various ideas have been put forth to effectively conceive the image analysis on various domain of them is a weight based colour transformation method for the microscopic images that takes the image specific colour 
content into account and can replace standard colour transform in compression schemes. One of the most promising feature extraction methods in recent years has been the region covariance used in image texture classification and object recognition. Region covariance method is extensively used in texture classification and recognition in image and videos.

Fractal coding is to find fractals which can best approximate the original image and then to represent them as asset of a fine transformations. It is always applied to gray level images, by splitting the RGB colour image into three channels, Red, Blue, Green process them and compress them separately. Out of the numerous methods proposed and developed one can be to determine the pixel's trichromatic coefficient within the homogenous blocks formed by hierarchical partitioning method. Instead of fractal coding here the concept of RGB plane separation with Wavelet Transformation is implemented.

\section{RELATED WORK}

Implementing the IRIS model onto an image is the most important part of the concept and then processing that image to obtain a suitable output in regards to various parameters, and that can be chrominance, bandwidth, compression ratio, time of computing, signal to noise ratio, error, etc.

The pathway is

- $\mathrm{RGB} \rightarrow$ Gray Plane

- Parameters identification

- Difference of Gaussian

- Activation degree

- Edge detection

- Discrete wavelet transform

- Inverse wavelet transform

- Performance parameters

The colour image is acquired and it is converted into the individual R, G, and B plane. The image is analysed individually to identify the parameters, then finding the difference of Gaussian value by ROC (Rank Order Coding). Thorpe et al $[1,2]$ proposed that the order in which spikes are emitted encodes for the stimulus.ROC relies on the following assumptions:

i. $\quad$ From stimulus onset, only the first spike emitted is considered in response.

ii. The time of fire of each ganglion cell is proportional to its degree of activation.

iii. Only the order of firing of the neuron encodes for the stimulus.

An $\mathrm{N}_{\mathrm{s}}$ layered grid G [2] of filters as an architecture for the retina model. Ganglion cells are regularly spaced by $2^{\mathrm{s}}$ pixels over the horizontal and Vertical axes. The set of ganglion cell location defines a subset $U$ of $\mathrm{N}^{3}$. For an $\mathrm{N}^{2}$ sized image, $U$ is defined by:

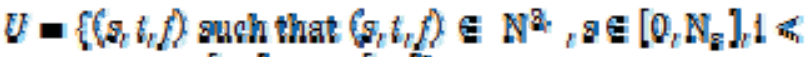 $\left.N_{t} f \sum_{i} N_{t} t \square\left[2^{z}\right] \& f \square\left[2^{2}\right]\right]$}

Where $s$ denotes the scale of the cell \& $(i, j)$ its position, then the response of a ganglion cell can be approximated by a linear filter. The dog filter [6] which is a weighted difference of Gaussian and is defined as follows:

$$
\operatorname{dog}(\mathrm{x}, \mathrm{y})=w_{c} \mathrm{~g}_{\sigma}(\mathrm{x}, \mathrm{y})-w_{s} \mathrm{~g}_{\alpha \sigma}(\mathrm{x}, \mathrm{y})
$$

where $\sigma$ is the central standard deviation of the filter, $\alpha$ is a priori fixed real number, $g_{\sigma}\left(\right.$ resp $\left.g_{\alpha \sigma}\right)$ is the Gaussian kernel of standard deviation $\sigma(\operatorname{resp} . \alpha \sigma)$ and $\mathrm{w}_{\mathrm{c}}\left(\right.$ resp. $\left.\mathrm{w}_{\mathrm{s}}\right)$ is the weight of $\mathrm{g}_{\sigma}\left(\right.$ resp. $\left.\mathrm{g}_{\alpha \sigma}\right)$. With biologically realistic parameters a dog filter applied to the image is a contour detector. So, in order to measure 
the degree of activation of a given neuron, we compute the convolution of the original image $\mathrm{f}$ by a dog filter defined by its scale s and location $(i, j)$ so that:

$$
C_{s i j=\Sigma} \sum_{y=-\alpha d o g}(i-x, j-y) f(x, y)
$$

Neuron responses are then sorted in the decreasing order of their amplitude, $\mathrm{i}: \mathrm{e}\left|\mathrm{C}_{\mathrm{sij}}\right|$.

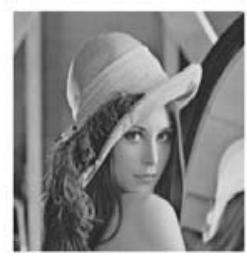

(a)

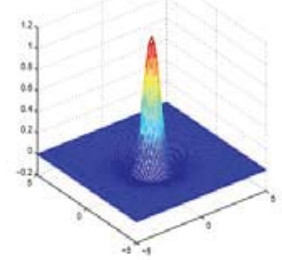

(b)

Figure 4: Behavior of the dog filter when applied to Lena (a) the classical test image Lena. (b) the dog filter with parameters $\alpha=3, w_{c}=w_{s}=1$ and $\sigma=0.5$. (c) The absolute value of the image resulting from the convolution of Lena with the filter in (b)

Edge detection is done using the Sobel, Canny, Prewitt, Robert, Log and Zero Cross cumulatively. It is important for image analysis and is used to identify areas where a large change in intensity occurs. In typical images, edge characterizes object boundaries and is useful for segmentation, registration and identification of objects in scene. There are three fundamental steps in edge detection:

- Image smoothing for noise reduction

- Detection of edge points

- Edge localization

Wavelet transform is an essential tool in image processing, it decomposes an image into a set of different resolution sub images, corresponding to the frequency bands, this results in a multi-resolution representation of images with localization in both spatial and frequency domains, this is not possible in case of Fourier and Cosine transforms which give good localization in one domain at the expense of other.

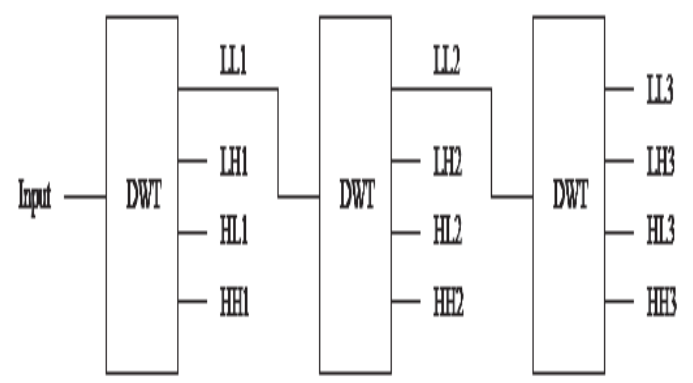

Fig 5: Three decomposition steps of an image using pyramidal decomposition

\section{SIMULATION/EXPERIMENTAL RESULTS}

In terms of the subjective quality, this method is superior to other conventional methods as it does not have a tendency to smooth the image, thereby losing texture and depth information. The compression ratio also is enhanced one and on an average it is up to $90 \%$ of the original image. The iris model implemented in biometric field enhances sorting and filtering of the image and the stimulus decoded is with an enhanced result. the 
proposed approach sets a way for the compression of a colour method image or gray image, gray image directly and colour image by segregation into the three respective planes $r, g$, $b$ which give an enhanced result after the iris model without rendering the important data, and compressing it with lesser complexity.

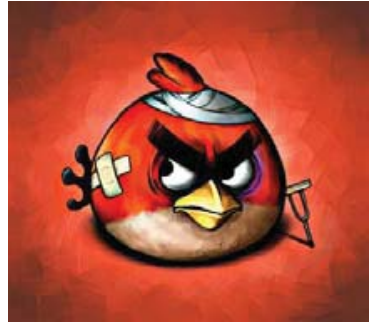

(a)

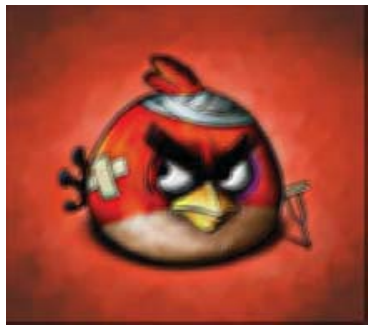

(b)

Fig. 6: Angry bird (a) Original image (b) Compressed image

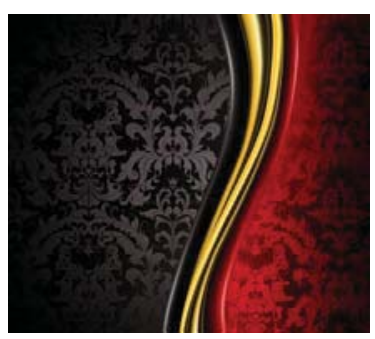

(a)

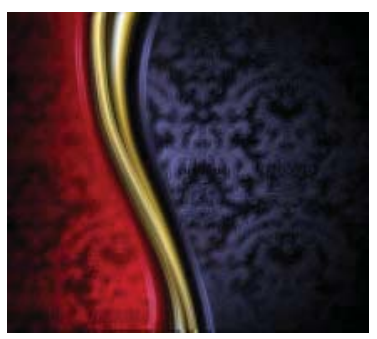

(b)

Fig. 7: Stripes (a) Original image, (b) Compressed image

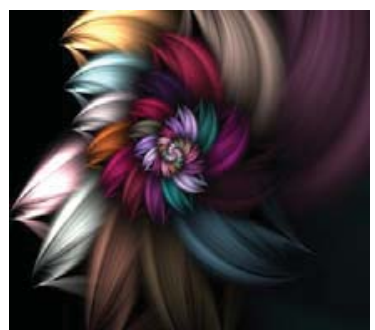

(a)

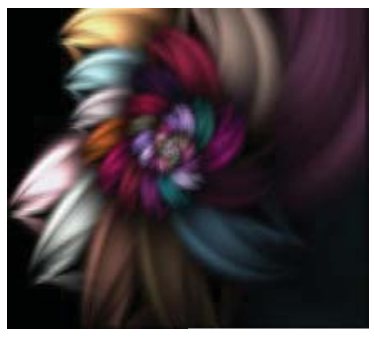

(b)

Fig. 8: Flower (a) Original image, (b) Compressed image

The Table shows the original size of a selected image and the size after compression of that image by our proposed IRIS model, it shows a significant reduction in size, which shows that our compression has a good impact and hence worthful.

Table 1. Compression Ratio and PSNR

\begin{tabular}{|l|l|l|l|}
\hline $\begin{array}{l}\text { S. } \\
\text { No. }\end{array}$ & Image Name & $\begin{array}{l}\text { Compression } \\
\text { Ratio (\%) }\end{array}$ & PSNR (Db) \\
\hline 1. & Angry Birds & 93.00 & 20.60 \\
\hline 2. & Stripes & 93.75 & 21.23 \\
\hline 3. & Flower & 93.75 & 22.69 \\
\hline
\end{tabular}




\section{. VIII. CONCLUSION}

Thus it can be concluded that the method gives a better output, the parameters evaluated are complementary for the image processing and can be utilized in some other domains also. This model and the parameters can be further enhanced to formulate newer dimension to bio inspired computing.

\section{REFERENCES}

[1] S. Thorpe, "Spike arrival times: A highly efficient coding scheme for neural networks," Parallel Processing in Neural Systems and Computers, pp. 91-94, 1990.

[2] R. Van Rullen and S. Thorpe, "Rate coding versus temporal order coding: What the retinal ganglion cells tell the visual cortex," Neural Computation, vol. 13, no. 6, pp. 1255-1283, June 2001.

[3] M. Antonini, M. Barlaud, P. Mathieu, and I. Daubechies, "Image coding using wavelet transform," IEEE Transactions on Image Processing, vol. 1, no. 2, pp. 205-220, April 1992.

[4] F. Rieke, D. Warland, R. de Ruyter van Steveninck, and W. Bialek, Spikes: Exploring the Neural Code, The MIT Press, Cambridge, MA, USA, 1997.

[5] T. Gollisch and M. Meister, "Rapid neural coding in the retina with relative spike latencies," Science, vol. 319, no. 5866, pp. 11081111, February 2008.

[6] Shaou-Gang Miaou, Chih-Lung Liu, “ A Quality-on-Demand Algorithm For Wavelet-Based Compression of Electrocardiogram Signals", IEEE Transactions on Biomedical Engineering, Vol. 49, No. 3, March 2002.

[7] B. Sen and S. Furber, "Maximising information recovery from rankorder codes," in Proceedings of SPIE conference on Data Mining, Intrusion Detection, Information Assurance, and Data Networks Security, Orlando, FL, U.S.A., April 2007, vol. 6570, p. 65700C.

[8] L. Perrinet, M. Samuelides, and S. Thorpe, "Coding static natural images using spiking event times: do neurons cooperate?," IEEE Transactions on Neural Networks, vol. 15, no. 5, pp. 1164-1175, September 2004.

[9] S.G. Mallat, "A theory for multiresolution signal decomposition : the wavelet representation," IEEE Transactions on Pattern Analysis and Machine Intelligence, vol. 11, no. 7, pp. 674-693, 1989.

[10] A. Wohrer and P. Kornprobst, "Virtual retina : A biological retina model and simulator, with contrast gain control," Journal of Computational Neuroscience, vol. 26, no. 2, pp. 219-249, 2009.

[11] Manuel Blanco-Velasco, Fernando Cruz-Roldan,Juan Ignacio Godino-Llorente andKenneth E. Barner, "Wavelet Packets Feasibility study for the Design of an ECG Compressor", IEEE Transactions on Biomedical Engineering, Vol. 54, No.4, April 2007.

[12] Oleksiy Pogrebnyak, Pablo Manrique Ramfrez, "Lifting-based Wavelet Transform Algorithm for Lossy Image/Signal Compression,"International conference on Electrical and Electronics Engineering ,2004.

[13] K. Masmoudi, M. Antonini, and P. Kornprobst, "Spike based neural codes : towards a novel bio-inspired still image coding schema," Tech. Rep. RR-7167, INRIA Spohia Antipolis-Mditerrane, January 2010.

[14] Khaled Masoudi, Marc Antonini, Pierre Kornprobst, Laurent Perrinet, "A Novel Bio- Inspired Static Image Comression Scheme For Noisy data Transmission Over Low-Bandwidth Channels.", The $35^{\text {th }}$ International Conference on Acoustics, Speech and Signal Processing (ICASSP), Dallas, United States, 2010 